\title{
Performance Analysis of Spatial Modulation-Multiple Input Multiple Output with Transmit Antenna Selection over Nakagami- $m$ Fading Channels
}

\author{
Aditya Sinha, Arnav Prateek, Rajesh R. \\ School of Electronics Engineering, VIT UNIVERSITY, Vellore, Tamil Nadu, India \\ publications850@gmail.com
}

\begin{abstract}
In this paper, the outage performance analysis of spatial modulation multiple input multiple output (SM-MIMO) system and transmit antenna selection (TAS) in Nakagami-m fading environment is proposed. The SM uses both the antenna indices as well as the signal constellation to transmit information bits. Further, TAS is employed in the proposed SM-MIMO to maximize the receive signal power. The closed form expression for the outage probability of the SM MIMO system with TAS for Nakagami-m channel is derived. Analytical results show that the outage performance of the proposed system with TAS is significantly better than SM without TAS.
\end{abstract}

Keyword - Spatial Modulation, Multiple input multiple output, Transmit antenna selection, Nakagami fading channels.

\section{INTRODUCTION}

Higher data rate and enhanced quality of service (QoS) are important factors in the wireless communication system and the most important points in 5G technology too [1]. The issues of incorporated reserve preparation within cellular networks are addressed in [2] and an enhancement of the proportional fairness algorithm (NIPFA) to examine the imminent $5 \mathrm{G}$ networks. Therefore it is very necessary to increase the bandwidth efficiency and the outage performance of the system. Multiple Input Multiple Output (MIMO) systems provide high data rates and enhanced system performance. MIMO system suffers from huge drawbacks such as it requires synchronization, since more number of antennas was used, the distance had to be maintained and there was inter channel interference (ICI). MIMO systems use parallel RF chains which increase the cost in terms of hardware complexity, power and size [3]. Vertical Bell Labs Layered Space-Time Architecture (V-BLAST) is a technique which increased the bandwidth efficiency but the problem of ICI persists. V-BLAST increases the receiver complexity and hence the system cost also increases. In V-BLAST, number of active transmitting antennas increases the number of RF chains which will increase the transmitting power, size and cost at the transmitter end. The receiver has to detect all the active transmitting antennas which will increase the receiver complexity. To solve the ICI problem and to provide high bandwidth efficiency while decreasing the system complexity and improving the error performance is spatial modulation (SM) [4].

The SM system uses only one transmit antenna which solved the problem of synchronization, enhanced the capacity and also improved efficiency. It requires only one RF chain reducing the complexity and cost [5]. It uses spatial and symbol constellation both to transmit data [6]. SM receiver estimates both transmitted symbol and transmit antenna index to decide the information bits. During transmission only one transmit antenna is active in the conventional SM. Besides the above advantages, SM receiver complexity is much lower than that of V-BLAST. Hence, SM has overall system complexity and cost advantages as well over conventional MIMO techniques.

Since only one antenna is active at the transmitting side, there is no transmit antenna diversity. However, by using "transmit antenna selection (TAS)" to the SM system, diversity can be achieved and BER performance can be further improved [7]. In literature, there are many studies on SM with TAS in Rayleigh Fading channel [7]-[11]. In [7], capacity optimized antenna selection (COAS) and Euclidean distance based antenna selection (EDAS) techniques are proposed for the SM and symbol error rate (SER) performance is given through Monte Carlo simulations. In [9], mathematical expression of outage probability is derived in Rayleigh fading channel for the SM system with COAS. In [10] and [11], new technique is proposed for Space Shift Keying (SSK) modulation which is a special case of SM. In this technique the symbol constellation is not used to carry information. Information is transmitted only through spatial constellation, antenna indices are used to carry information. 
In the recent studies on SM and SSK techniques, bit error rates and outage performance are analysed for more general fading channels [12]-[14], such as Nakagami- $m$ channels. There is no diversity gain for systems without antenna selection even if $m$ is varied. In [14] it is shown that to obtain diversity gain in Nakagami$m$ channels, the use of TAS techniques in the classical SM and SSK systems can be done.

In this paper the outage probability of SM MIMO with TAS in Nakagami- $m$ fading channels is analyzed for arbitrary value of $m$. A closed form analytical term for the outage probability is derived. Analytical results show that the outage performance of the proposed system significantly improved by TAS compared to conventional system of SM without TAS.

The rest of the paper is organized as follows. In Section II, the system model considered is described. In Section III, the closed-form outage probability expression of the system is obtained. The closed-form expression for a special condition of Nakagami- $m$ channel is also obtained. Comparisons of computer simulation and analytical results are given in Section IV. Finally, Section VI concludes the paper.

\section{SYSTEM MODEL}

Consider a point to point communication system model between Node A and Node B. It is assumed that both the nodes are equipped with $M_{t}$ transmit antennas and $M_{r}$ receive antennas as shown in the Fig. 1 . In this proposed work $M_{S}$ transmit antennas are selected which maximize the received SNR at the receiver from the available $M_{t}$ antennas.

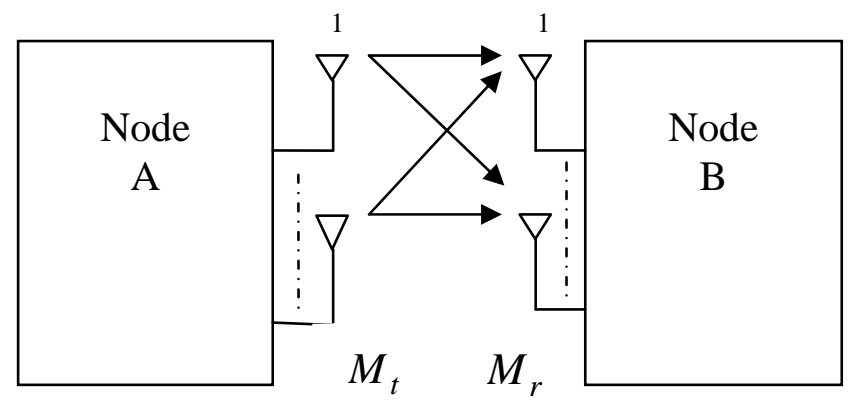

Fig. 1 System model of SM-MIMO with antenna selection

The information bits are mapped to the selected $M_{S}$ antennas and modulated symbols. $M_{S}$ should be a power of two since mapping is based on the binary information bits in SM. It is assumed that perfect channel state information (CSI) is available at the receiver and the transmitter doesn't require prior knowledge of the channel. At the receiver, there are $M_{r}$ receive antennas and maximum ratio combiner (MRC) is used. The received signal vector $\mathbf{y}$ is given as

$$
\mathbf{y}=\mathbf{h}_{\ell} s+\mathbf{w}
$$

Where $s$ is the information symbol transmitted from transmitter $\mathrm{S}_{1}, \mathbf{w}$ is the additive Gaussian noise vector. Also, vector $\mathbf{h}_{\ell}$ is the $\ell$ th column of $M_{r} \times M_{S} \mathbf{H}$ channel matrix $\left(\ell=1,2, \ldots, M_{S}\right)$, so $\mathbf{h}_{\ell}$ is the channel coefficient vector between the $\ell$ th transmit antenna and receive antennas and it can be given as

$$
\mathbf{h}_{\ell}=\left[h_{1, \ell}, h_{2, \ell}, \ldots, h_{M_{r}, \ell}\right]^{T}
$$

Here, complex channel coefficient $h_{i, \ell}\left(i=1, \ldots, M_{r}\right)$ is given as $h_{i, \ell}=h_{i, \ell, I}+j h_{i, \ell, Q}=\left|h_{i, \ell}\right| e^{j \theta_{i, \ell}}$ where $\left|h_{i, \ell}\right|$ denotes amplitude and $\theta_{i, \ell}$ denotes phase. The detector on the receiver side has ideal state channel information (CSI) detecting the transmit antenna index, $\ell$, and information signal $s$, with the ML decision rule as follows

$$
[\hat{\ell}, \hat{s}]=\arg _{\ell, s} \min \left\|\mathbf{y}-\mathbf{h}_{\ell} s\right\|
$$

The PDF of Nakagami- $m$ distribution is given by

$$
f_{A_{j}}(r)=\frac{2}{\Gamma(m)}\left(\frac{m}{\Omega}\right)^{m} r^{2 m-1} e^{\frac{-m r^{2}}{\Omega}}, \forall i, \ell
$$


where $m$ is the fading parameter, $\Gamma($.$) is gamma function [17, (8.310.1)]. Also, \Omega$ is defined as $\Omega=E\left[\left|h_{i, \ell}\right|^{2}\right], \forall i, \ell$.In the proposed work $\Omega=1$ is assumed. The phase of the channel coefficients is statistically between $(0,2 \pi)$ of the random variables which are independent of each other and are uniformly distributed.

In proposed technique, antenna selection is based on the maximization of norms of the channel coefficients. Norm square of the channel coefficients for the $\ell^{\text {th }}$ transmit antenna can be given as

$$
A_{j}=\left\|\mathbf{h}_{\ell}\right\|^{2}=\sum_{i=1}^{M_{r}}\left|h_{i, \ell}\right|^{2}
$$

where $A_{j}$ is the chi-squared distributed random variable with $2 m M_{r}$ degrees of freedom. The norms of channel coefficients between transmit and receive antennas are ordered in an ascending order. $M_{S}$ transmit antennas corresponding to the largest channel norms are selected from $M_{t}$ transmit antennas and SM is applied to the selected transmit antennas. Bandwidth efficiency of the system, in terms of $b i t s / s / H z$, is $\varsigma=\frac{R_{b}}{B}=\log _{2} M_{S}+\log _{2} G$ where $R_{b}$ data rate is, $B$ is bandwidth. Also, $G$ represents the symbol constellation size. As seen, the bandwidth efficiency increases as the number of selected antennas $M_{S}$ and/or constellation size $G$ increases.

\section{OUTAGE PROBABILITY ANALYSIS}

In this section, analytical expressions for the outage probability are derived for the proposed network. The outage probability that the instantaneous end-to-end received $\operatorname{SNR}\left(\gamma_{\text {end }}\right)$ is lower than a predetermined threshold $\operatorname{SNR}\left(\gamma_{\text {th }}\right)$ defined as

$$
P_{\text {out }}=P_{r}\left\{\gamma_{\text {end }}<\gamma_{\text {th }}\right\}=\int_{0}^{\gamma_{\text {th }}} f_{\gamma_{\text {end }}}(r) d r
$$

where $f_{\gamma_{\text {end }}}(x)$ is the PDF of the instantaneous received SNR. In order to simplify the analysis, we assume that the antenna index error at the receiver is negligible. It is known that this is a good approach to determine receiver performance of the SM systems when the constellation size $G$ is sufficiently more than the numbers of transmit antennas [7]. Therefore $\gamma_{t h}$ is defined as $\gamma_{t h}=\frac{2^{C-\log _{2} M_{s}-1}}{S N R}$ for a given capacity $C$. As seen from (6), $f_{\gamma_{\text {end }}}(x)$ is required to obtain the outage probability of the system. In this section, PDF of the instantaneous received SNR which belongs to the SM scheme with COAS is obtained in Nakagami- $m$ fading channels and the closed-form exact outage probability is derived for arbitrary $m$.

In Nakagami- $m$ MIMO channel norm square of $\left\|\mathbf{h}_{\ell}\right\|^{2}, \ell=1,2, \ldots, M_{t}$ is independently and identically distributed chi-squared random variable with $2 m M_{r}$, degrees of freedom. Hence PDF is given by [14]

$$
f_{A_{j}}(r)=\frac{1}{2^{m N_{r}} \Gamma\left(m M_{r}\right)\left(\frac{1}{2 m}\right)^{m M_{r}}} r^{m M_{r}-1} e^{-m M_{r}}, r \geq 0
$$

Also the CDF is calculated as [15]

$$
F_{A_{j}}(r)=1-e^{-m r} \sum_{k=0}^{m M_{r}-1} \frac{(m r)^{k}}{k !}, r \geq 0
$$

where $\gamma(.,$.$) is the lower incomplete gamma function [17, (8.350.1)]. As per order statistics PDF of the random$ variable $\left\|\mathbf{h}_{\ell}\right\|^{2}$ where $\left\|\mathbf{h}_{(1)}\right\|^{2}<\left\|\mathbf{h}_{(2)}\right\|^{2}<\ldots<\left\|\mathbf{h}_{(s)}\right\|^{2}<\ldots<\left\|\mathbf{h}_{\left(M_{t}\right)}\right\|^{2}$ can be given as [16, (2.1.6)]

$$
f_{A_{(r)}}(r)=\frac{1}{B\left(s, M_{t}-s+1\right)}\left[F_{A_{j}}(r)\right]^{s-1}\left[1-F_{A_{j}}(r)\right]^{M_{t}-s} f_{A_{j}}(r)
$$


Where $c=M_{t}-M_{S}+1$ and $B(.,$.$) is the beta function [17, (8.380.1)]. By averaging the PDF's of the received$ SNRs, PDF of the instantaneous SNR can be written as [8]

$$
\begin{aligned}
f_{\gamma_{i}}^{c}(r)= & \frac{1}{\left(M_{t}-c+1\right)} \sum_{n=c}^{M_{t}} \frac{1}{B\left(n, M_{t}-n+1\right)}\left\{1-e^{-m r} \sum_{k=0}^{m M_{r}-1} \frac{(m r)^{k}}{k !}\right\}^{i-1}\left\{e^{-m r} \sum_{k=0}^{m M_{r}-1} \frac{(m r)^{k}}{k !}\right\}^{M_{t}-i} \\
& \times \frac{m^{m M_{r_{r}}{ }^{m M-1} e^{-m r}}}{\Gamma\left(m M_{r}\right)}
\end{aligned}
$$

Using Binomial expansion $\left[1-e^{-m M_{r}} \sum_{k=0}^{m M_{r}-1} \frac{(m r)^{k}}{k !}\right]^{n-1}$ is defined as

$$
\left[1-e^{-m M_{r}} \sum_{k=0}^{m M_{r}-1} \frac{(m r)^{k}}{k !}\right]^{n-1}=\sum_{i=0}^{n-1}(-1)^{i}\left(\begin{array}{c}
n-1 \\
i
\end{array}\right)^{i}\left[e^{-m r} \sum_{k=0}^{m M_{r}-1} \frac{(m r)^{k}}{k !}\right]
$$

Substituting the above equation (11) the PDF of instantaneous SNR is given as

$$
\begin{aligned}
f_{\gamma_{i}}^{c}(r) & =\frac{m^{m M_{r}}}{\left(M_{t}-c+1\right) \Gamma\left(m M_{r}\right)} \sum_{n=c}^{M_{t}} \sum_{i=0}^{n-1} \sum_{j=0}^{N} \frac{1}{B\left(n, M_{t}-n+1\right)}\left(\begin{array}{c}
n-1 \\
i
\end{array}\right)(-1)^{i} Q_{j}\left(i, m M_{r}\right) \\
& \times e^{-m r\left(M_{t}-n+i+1\right)_{r} m M_{r}-1+j}
\end{aligned}
$$

where $N=\left(m M_{r}-1\right)\left(M_{t}-n+i\right)$ and $Q_{j}\left(i, m M_{r}\right)$ is the coefficient of $r^{k}$ in the expansion of

$$
\left[\sum_{k=0}^{m M_{r}-1} \frac{(m r)^{k}}{k !}\right]^{M_{t}-n+i}
$$

The outage probability for a SM-MIMO system with TAS is given as

$$
\begin{aligned}
P_{\text {out }}= & \frac{m^{m M_{r}}}{\left(M_{t}-c+1\right) \Gamma\left(m M_{r}\right)} \sum_{n=c}^{M_{t}} \frac{1}{B\left(n, M_{t}-n+1\right)} \sum_{i=0}^{n-1}\left(\begin{array}{c}
n-1 \\
i
\end{array}\right)(-1)^{i} \sum_{j=0}^{N} Q_{j}\left(i, m M_{r}\right) \\
& \times \sum_{i=0}^{n-1}\left(\begin{array}{c}
n-1 \\
i
\end{array}\right)(-1)^{i} \sum_{j=0}^{N} Q_{j}\left(i, m M_{r}\right) \int_{0}^{\gamma_{t h}} e^{-m r\left(M_{t}-n+i+1\right)} r_{r}^{m M_{r}-1+j} d r
\end{aligned}
$$

The above integral (14) is calculated using[17, (3.944.3)], and the outage probability expression for SM-MIMO with TAS system is given as

$$
\begin{aligned}
P_{\text {out }}= & \frac{m^{m M_{r}}}{\left(M_{t}-c+1\right) \Gamma\left(m M_{r}\right)} \sum_{n=c}^{M_{t}} \frac{1}{B\left(n, M_{t}-n+1\right)} \sum_{i=0}^{n-1}\left(\begin{array}{c}
n-1 \\
i
\end{array}\right)(-1)^{i} \sum_{j=0}^{N} Q_{j}\left(i, m M_{r}\right) \\
& \times \frac{\gamma\left(m M_{r}+j, m \gamma_{t h}\left(M_{t}-n+i+1\right)\right)}{\left(M_{t}-n+i+1\right)^{m M_{r}+j}}
\end{aligned}
$$

Where $\gamma(.,$.$) is the lower incomplete gamma function. The closed-form outage probability for the Rayleigh$ channel $\quad(m=1)$ is given as follows for

$$
\begin{aligned}
P_{\text {out }}(\gamma, R)= & \frac{1}{\left(M_{t}-c+1\right) \Gamma\left(M_{r}\right)} \sum_{n=c}^{M_{t}} \frac{1}{B\left(n, M_{t}-n+1\right)} \sum_{i=0}^{n-1}\left(\begin{array}{c}
n-1 \\
i
\end{array}\right)(-1)^{i} \\
& \times \sum_{j=0}^{N} Q_{j}\left(i, M_{r}\right) \frac{\gamma\left(M_{r}+j, \gamma t h\left(M_{t}-n+i+1\right)\right)}{\left(M_{t}-n+i+1\right)^{M_{r}+j}}
\end{aligned}
$$


The outage probability of the SM MIMO systems without TAS in the Nakagami- $m$ fading channels, $P_{\text {out }}(\gamma R)_{\text {withoutTAS }}$ can be given as

$$
P_{\text {out }}(\gamma, R)_{\text {SMwithoutTAS }}=\frac{1}{2^{m M_{r}} \Gamma\left(m M_{r}\right)\left(\frac{1}{2 m}\right)^{m M_{r}}} \int_{0}^{\gamma_{t h}} r M_{r}-1 e^{-m M_{r}} d r
$$

The above integral (17) is calculated using [17, (3.944.3)], the outage probability for a SM-MIMO system without TAS is given as

$$
P_{\text {out }}(\gamma, R)_{\text {SMwithoutTAS }}=1-e^{-m \gamma_{t h}} \sum_{i=0}^{m M_{r}-1} \frac{\left(m \gamma_{t h}\right)^{i}}{i !}
$$

\section{NUMERICAL RESULT}

In this section, the outage performance of the proposed SM-MIMO with TAS and SM-MIMO without TAS are compared using the analytical expressions derived in Section III. The list of parameters for the outage analysis is listed in Table I.

Table. I List of Parameters

\begin{tabular}{|c|c|}
\hline Parameters & Values \\
\hline Data Rate $\left(R_{b}\right)$ & $1-6 \mathrm{bits} / \mathrm{s} / \mathrm{Hz}$ \\
\hline SNR at source nodes A and B (SNR) & $-5 \mathrm{~dB}-30 \mathrm{~dB}$ \\
\hline Total number of transmit antennas at source node A $\left(M_{t}\right)$ & $4,6,8,10$ \\
\hline Number of transmit antennas selected at source node A & 2 \\
\hline Number of antennas at Node B & 3 \\
\hline
\end{tabular}

The outage performance of the proposed SM-MIMO point to point network is shown in Fig. 2 at a data rate of $R_{b}=2 \mathrm{bits} / \mathrm{s} / \mathrm{Hz}$ in Nakagami- $m$ fading environment for the various values of fading parameter $m=0.5,1,2,3$ for $M_{t}=4, M_{s}=2$ and $M_{r}=3$. It is observed that at an outage probability of $10^{-2}$ for the proposed system, the minimum SNR requirement is around $3 \mathrm{~dB}$ when $m=0.5$. As the fading parameter is increased to 1,2 and 3 the minimum SNR requirement is around $-2 \mathrm{~dB},-3 \mathrm{~dB}$ and $-2.75 \mathrm{~dB}$ respectively. Whereas in the case of conventional SM MIMO system without TAS the minimum SNR requirement is $22 \mathrm{~dB}$. A significant improvement in the outage probability is observed for the proposed system over conventional SM MIMO system without TAS by increasing the fading parameter.

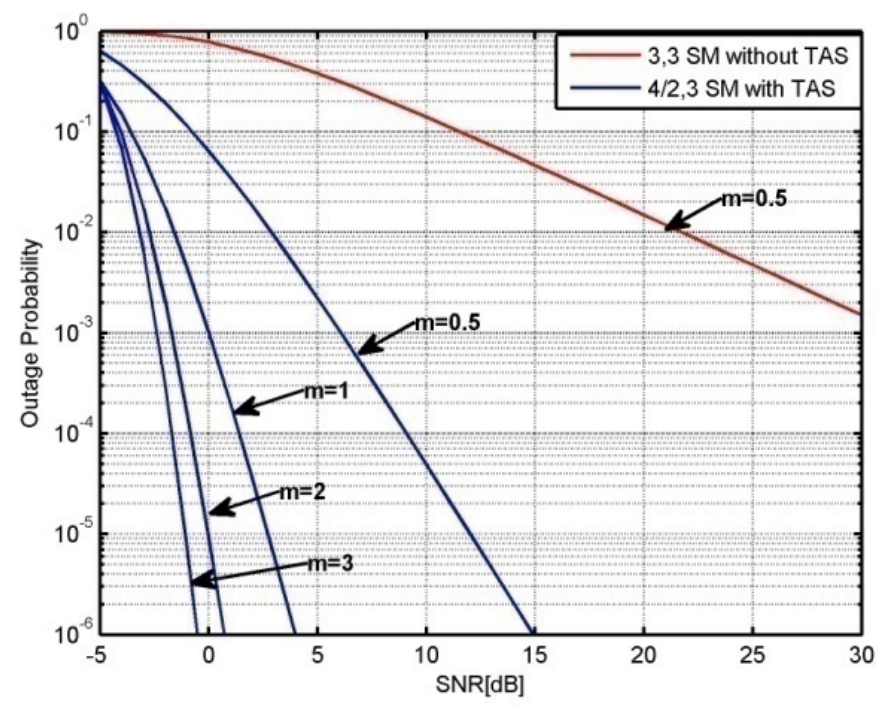

Fig.2 Outage probability against SNR curve for SM systems with arbitrary m values (2bits/s/Hz) 
Fig. 3 the outage performance of the proposed SM MIMO point to point system in Nakagami- $m$ fading channel for $M_{t}=4,6,8,10, M_{s}=2, M_{r}=3$ and $m=1$ is simulated. In Fig. 3 the outage performance of the proposed SM MIMO system with TAS is shown for a transmit antenna diversity. It is observed at an outage probability of $10^{-1}$ for $M_{t}=4, M_{s}=2, M_{r}=3$ and $m=1$ the minimum SNR requirement is $1 \mathrm{~dB}$. Also, it is observed that the increase in the number of transmit antenna available at Node A to 6, 8, 10 increases the outage probability with SNR requirement of $0 \mathrm{~dB},-1.5 \mathrm{~dB}$ and $-1 \mathrm{~dB}$. In the case of SM without TAS, the outage probability increases to $8 \mathrm{~dB}$.

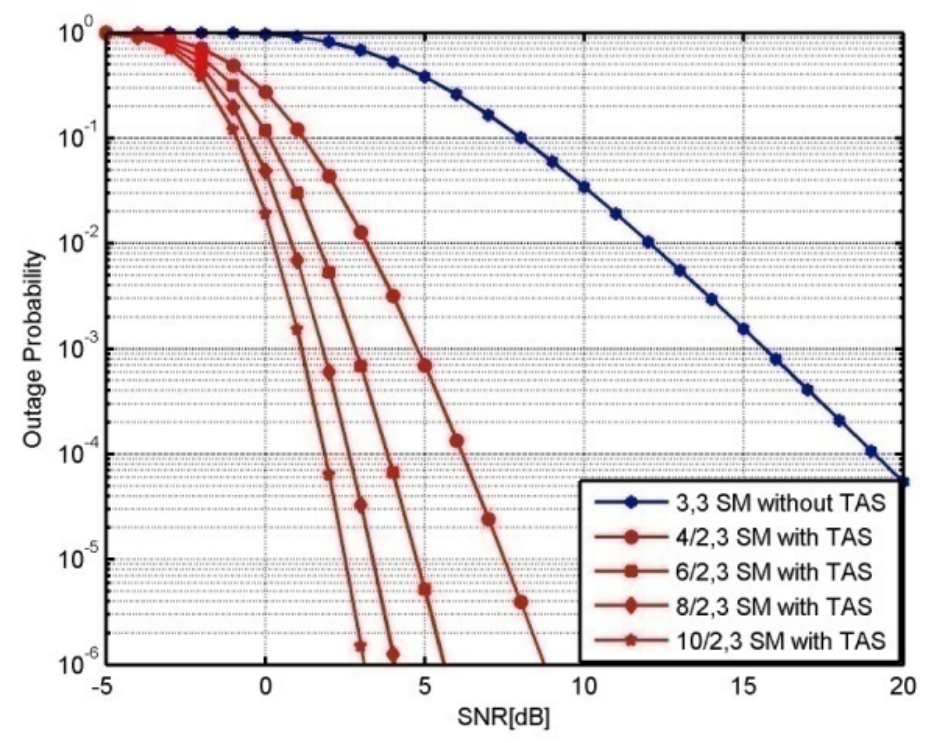

Fig.3 Outage probability against SNR curve for SM systems with m=1 (3bits/s/Hz)

In Fig. 4the outage performance of the proposed SM MIMO with TAS and without TAS is shown for varying rate $R_{b}$ from $1 \mathrm{bits} / \mathrm{s} / \mathrm{Hz}$ to $6 \mathrm{bits} / \mathrm{s} / \mathrm{Hz}$. The number of antennas at the source nodes is assumed to be $M_{t}=4,6,8,10, M_{s}=2, M_{r}=3, m=2$ and SNR=5 dB. It is observed for $M_{t}=4$ at an outage probability of $10^{-1}$, the data rate is $4.2 \mathrm{bits} / \mathrm{s} / \mathrm{Hz}$. As the number of antennas at the source nodes increases to 6, 8 and 10 for the same outage probability of $10^{-1}$ the data rate increases to $4.4 \mathrm{bits} / \mathrm{s} / \mathrm{Hz}, 4.5 \mathrm{bits} / \mathrm{s} / \mathrm{Hz}$ and $4.6 \mathrm{bits} / \mathrm{s} / \mathrm{Hz}$ respectively. In the case of SM MIMO without TAS the data rate is $2.6 \mathrm{bits} / \mathrm{s} / \mathrm{Hz}$. It is observed that the increase in the number of antennas the source nodes increases the data rate significantly for the proposed system compared to SM MIMO without TAS.

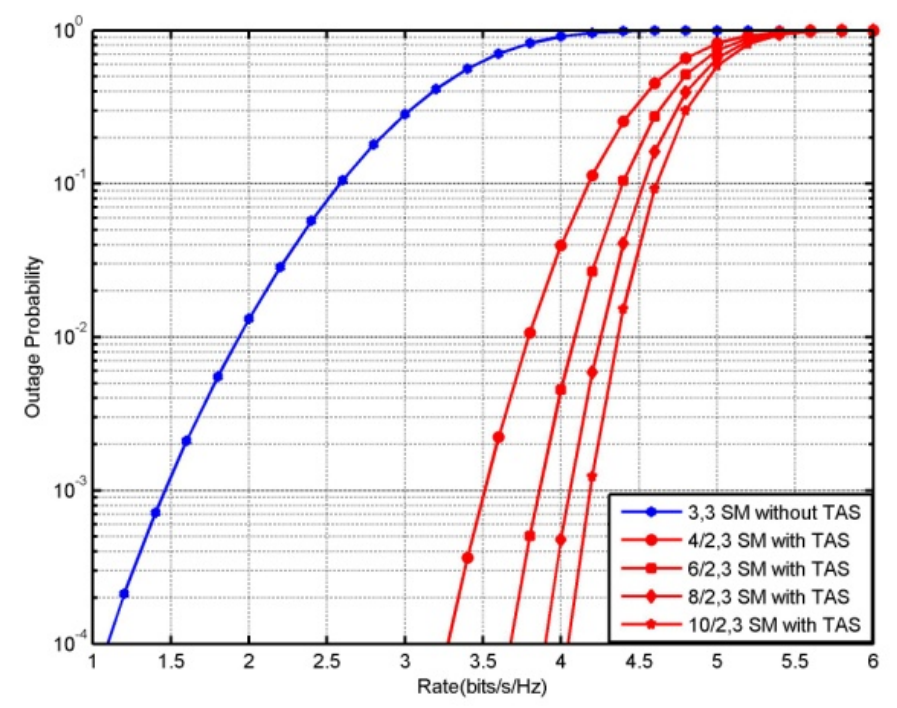

Fig.4 Outage probability against Rate curve for SM systems with $m=2(S N R=5 d B)$ 


\section{CONCLUSION}

In this paper the outage performance of the SM MIMO with TAS in point-to-point network is proposed to maximise the received signal power. A closed form analytical expression is derived for the end-to-end outage probability of the proposed system in Nakagami- $m$ fading channel environment. The outage performance of the proposed network shows a significant improvement over the conventional SM without TAS at the rate of increase of antennas at the source nodes and also by varying $m$.

\section{IV.REFERNCES}

[1] Rupendra Nath Mitra, Dharma P. Agrawal, “5G mobile technology: A survey,” ICT Express 1,pp. 132-137, 2015

[2] Abubakar M. Miyim; Mahamod Ismail; Rosdiadee Nordin, "Enhanced cellular systems for cooperative communication in 5G networks,” IEEE Conference Publications, 2016, pp. 440 - 444

[3] Sanayei, S., and Nosratinia, A.: 'Antenna selection in MIMO systems’, IEEE Commun. Mag., 2004, 42, pp. 68-73

[4] R. Mesleh, H. Haas, C.W. Ahn, and S. Yun, "Spatial modulation - a new low complexity spectral efficiency enhancing technique," Commun. Netw. China, pp. 1-5 Oct. 2006.

[5] M. D. Renzo, H. Haas, A. Ghrayeb, S. Sugiura and L. Hanzo, "Spatial modulation for generalized MIMO: challenges, opportunities, and Implementation," Proc. IEEE, vol. 102, no. 1, pp. 56-103, Jan. 2014.

[6] J. Jeganathan, A. Ghrayeb, L. Szczecinski and A. Ceron, "Space shift keying modulation for MIMO channels," IEEE Trans. on Wireless Commun., vol. 8, no. 7, pp. 3692-3703, Jul. 2009.

[7] R. Rajashekar, K. V. S. Hari, and L. Hanzo, “Antenna selection in spatial modulation systems,” IEEE Commun. Let., vol. 17, no. 3, pp. 521-524, Mar. 2013.

[8] N. Pillay, and H. Xu, “Comments on 'Antenna selection in spatial modulation systems'," IEEE Commun. Let., vol. 17, no. 9, pp. 1681-1683, Sep. 2013.

[9] B. Kumbhani, and R.S. Kshetrimayum, "Outage probability analysis of spatial modulation systems with antenna selection," IET Elect. Let., vol. 50, no. 2, pp. 125-126, Jan. 2014.

[10] E. Zhou, and L. Hao, "Transmit antenna selection in space shift keying modulation," IEEE 77th Veh. Technol. Conf. (VTC Spring), Jun. 2013.

[11] K. Ntontin, M. D. Renzo, A. Perez-Neira, and C. Verikoukis, "Performance analysis of antenna subset selection in space shift keying systems," in Proc. IEEE CAMAD Conf., Sept. 2013, pp.154-158.

[12] M. D. Renzo, and H. Haas, "A general framework for performance analysis of space shift keying (SSK) modulation for MISO correlated nakagami-m fading channels," IEEE Trans. on Commun., vol. 58, no. 9, pp. 2590-2603, Sept. 2010.

[13] M. D. Renzo, and H. Haas, "Bit error probability of SM-MIMO over generalized fading channels," IEEE Trans. on Veh. Technol., vol. 61, no. 3, pp. 1124-1144, Mar. 2012.

[14] M. D. Renzo, and H. Haas, "Bit error probability of space modulation over nakagami-m fading: asymptotic analysis," IEEE Commun. Let., vol. 15, no. 10, pp. 1026-1028, Oct. 2011.

[15] J. G. Proakis and M. Salehi, Digital Communications, 5th ed. New York: McGraw-Hill, 2008.

[16] H. A. David, and H. N. Nagaraja, Order Statistics, 3rd ed. New York: Wiley Interscience, 2003.

[17] S. Gradshteyn, and I. M. Ryzhik, Table of Integrals, Series, and Products, 7th ed. New York: Academic Press, 2007. 